\title{
Stability of CDFEs With Multiple Known and Unknown Delays: Discretized LKF Approach
}

\author{
Hongfei Li \\ Department of Mathematics, Shaanxi Xueqian Normal University, Xi' an 710100, China. \\ E-mail: 1hf8165@163.com
}

\begin{abstract}
This article considers the stability problem of a class of coupled differential-Functional equations(CDFEs) with multiple delays via the discretized Lyapunov-Krasovskii functional (LKF) approach. The system discussed contains both distributed delays known and discrete delays unknown, a LKF with two parts is chosen, where one part is a complete quadratic LKF which involves those known delays only and can be done discretization, the other is a simple LKF for the unknown delays. Through independently divided every known delay region that the plane regions consists in two known delays to discritized the LKF, the stability conditions of the systems are established based on a linear matrix inequality (LMI), which is dependent with the known delays, is independent with the unknown. Finally, Two examples are given to illustrate that the result is reliable and effective.
\end{abstract}

Keywords-discretized LKF approach; coupled differentialfunctional equations; multiple unknown discrete delays; multiple known distributed delays

\section{INTRODUCTION}

The coupled differential-functional equations

$$
\begin{aligned}
\dot{x}(t) & =f\left(t, x(t), y_{t}\right), \\
y(t) & =g\left(t, x(t), y_{t}\right)
\end{aligned}
$$

offers significant advantage over the traditional model of retarded and neutral type. Typically, the number of state variables in the practical systems may be rather large, but only a few components involve delay elements and are often low dimensional, i.e. $\operatorname{dim}(x(t)) \gg \operatorname{dim}(y(t))$. It is well known that many electrical, fluid and other systems initially described by partial differential equations can be arrived at a set of coupled differential-difference equations(CDDEs) as lossless propagation model [1][2], steam pressure control problem and water transmission problem [3]. Up to early 1990s, such systems were analyzed by taking the systems in a class of standard DDEs of neutral type. A nice overview is to analysis it directly as [4]. Related to most important works can be found in the references [5][6][7]. Gu and Niculescu [5] showed the existence of a quadratic LKF is necessary and sufficient for the stability of linear CDDE with single delay. Gu and Liu [9] generalized the difference equations to functional equations under the assumption of input-to-output stability (IOS) of the equations, and considered the uniform asymptotic stability of the systems. [6] also formulated a discretized LKF method based on the

\footnotetext{
${ }^{0}$ This work has been supported by Nature Science Foundation of Shaanxi Province under Grant 2011JM1009 and The Specialized Fund for the HighLevel Talent, Shaanxi Xueqian Normal University(No.2014DS019).
}

quadratic functional in [5]. $\mathrm{Gu}$ [7] extend the formulation of single delay to the case of multiple delay channels, and [10] generalized the analytical results of [5][6].

\section{A special form of CDFEs}

$$
\begin{aligned}
& \dot{x}(t)=f_{0}\left(t, x(t), y_{t}\right)+\int_{-r}^{0} H(\sigma) y(t+\sigma) \mathrm{d} \sigma, \\
& y(t)=g_{0}\left(t, x(t), y_{t}\right)+\int_{-r}^{0} M(\sigma) y(t+\sigma) \mathrm{d} \sigma
\end{aligned}
$$

are more general than CDDEs. Specially, $H(\sigma)$ and $M(\sigma)$ in $[-r, 0)$ may be bounded, piecewise constant matrices. Such a system may be written as a forward system, and

$$
u_{i}(t)=K_{i} \int_{-r_{i}}^{0} y(t+\theta) \mathrm{d} \theta, i=1,2, \ldots, N
$$

as the feedback. Closely, Li [11] discussed the stability of CDFEs with a discrete and distributed delays via discretized LKF approach. In engineering practice, however, some of the time-delays for the systems are known or can be measured in a finite region, the others are difficult to measure, even if they can be estimated, is also very big. So we hope to control known delays in input to stabilizing system. In this paper we will investigate the stability of such CDFEs with multiple known and unknown discrete delays and known distributed delays. And we wish to set up the stability condition with a well robustness, which is dependent with the known or small delays, and is independent with those unknown or large delays.

In this article, $\mathbb{R}^{n}$ and $\mathbb{R}^{m \times n}$ represent the set of real $n$ vectors and $m$ by $n$ matrices, respectively. $\mathbb{R}_{+}$denote the sets of positive real numbers. For a given delays $r_{i} \in \mathbb{R}_{+}(i=$ $1,2, \ldots, N), \mathcal{P C}\left([-r, 0), \mathbb{R}^{m}\right)$ is the space of bounded, right continuous, and piecewise continuous functions defined on $[-r, 0)\left(r=\max _{1 \leq i \leq N}\left\{r_{i}\right\}\right)$. These spaces are equipped with the $L_{2}$ norm $\|\phi\|_{L_{2}}^{2}=\int_{-r}^{0}\|\phi(\theta)\|^{2} \mathrm{~d} \theta$. For a given function $y(t) \in \mathbb{R}^{m}$ and $\sigma \in \mathbb{R}$, we define $y_{\sigma} \in \mathcal{P C}$ by $y_{\sigma}(\theta)=y(\sigma+\theta)$, $\theta \in[-r, 0)$. $\|\cdot\|$ denotes 2 -norm for vectors and matrices. $\lambda_{\min }(M)$ denotes the minimum in eigenvalues of Matrix $M$. $\left(R_{i j}\right)_{i=i_{1} \rightarrow i_{m}}^{j=j_{1} \rightarrow j_{n}}$ denotes a block matrix with some block matrices $R_{i_{k} j_{l}}$, where the line index $i$ is from $i_{1}$ to $i_{m}$ and the column index $j$ is from $j_{1}$ to $j_{n}$. 


\section{Problem Statement}

In this section, we discuss the system described by the following coupled functional-differential equations

$$
\begin{aligned}
& \dot{x}(t)=A x(t)+\sum_{i=1}^{N+M} B_{i} y\left(t-r_{i}\right)+\sum_{i=1}^{N} H_{i} \int_{-r_{i}}^{0} y(t+\theta) \mathrm{d} \theta, \\
& y(t)=C x(t)+\sum_{i=1}^{N+M} D_{i} y\left(t-r_{i}\right)+\sum_{i=1}^{N} M_{i} \int_{-r_{i}}^{0} y(t+\theta) \mathrm{d} \theta
\end{aligned}
$$

with the initial condition

$$
x\left(t_{0}\right)=\psi, \quad y_{t}=\phi .
$$

where $x \in \mathbb{R}^{n}, y \in \mathbb{R}^{m}$ are state vectors, $r_{i} \in \mathbb{R}_{+}$ $(i=1,2, \ldots, N)$ is some known delays, $r_{N+j} \in \mathbb{R}_{+}(j=$ $1,2, \ldots, M)$ is some unknown delays and $r=\max _{1<i<N+M}\left\{r_{i}\right\}$, $A \in \mathbb{R}^{n \times n}, C \in \mathbb{R}^{m \times n}, B_{i}, H_{i} \in \mathbb{R}^{n \times m}, D_{i}, M_{i} \in$ $\mathbb{R}^{m \times m}, i=1,2, \ldots, K$, are some known real matrices.

The Lyapunov-Krasovskii functional method is an effective method of studying the stability of a system described by differential-functional equations. Choose the LKF as

$$
V\left(x(t), y_{t}\right)=V_{1}+V_{2},
$$

where

$$
\begin{aligned}
V_{1}= & x^{T}(t) P x(t)+2 x^{T}(t) \sum_{i=1}^{N} \int_{-r_{i}}^{0} Q_{i}(\eta) y(t+\eta) \mathrm{d} \eta \\
& +\sum_{i=1}^{N} \sum_{j=1}^{N} \int_{-r_{i}}^{0} \int_{-r_{j}}^{0} y^{T}(t+\xi) R_{i j}(\xi, \eta) y(t+\eta) \mathrm{d} \eta \mathrm{d} \xi \\
& +\sum_{i=1}^{N} \int_{-r_{i}}^{0} y^{T}(t+\xi) S_{i}(\xi) y(t+\xi) \mathrm{d} \xi \\
V_{2}= & \sum_{j=N+1}^{N+M} \int_{-r_{j}}^{0} y^{T}(t+\xi) \bar{S}_{j} y(t+\xi) \mathrm{d} \xi .
\end{aligned}
$$

Remark 1: In the $\operatorname{LKF}(3), V_{1}$ is a completely quadratic LKF which may be manipulate to discretizated and respect only to the known delays , $V_{2}$ is a simple LKF and relate to those unknown or large delays.

For the LKF of (3), it is no difficult to induce the following proposition by Theorem 3 in [6].

Proposition 2: Suppose the difference-integral equation (DIE) of (2) is input-to-output stable. If there exist symmetric positive definite matrix $P$, and continuous matrix functions $Q_{i}(\eta), S_{i}(\eta)$ on $\left[-r_{i}, 0\right)$ and $R_{i j}(\xi, \eta)=R_{j i}^{T}(\xi, \eta)$ on $\left[-r_{i}, 0\right) \times\left[-r_{j}, 0\right)(\mathrm{i}, \mathrm{j}=1,2, \ldots, \mathrm{N})$ and $\bar{S}_{i}$ on $\left[-r_{N+k}, 0\right)(k=$ $1,2, \ldots, M)$ such that the LKF of (3) satisfy

$$
\begin{aligned}
V\left(x(t), y_{t}\right) & \geq \varepsilon\|x(t)\|^{2}, \\
\dot{V}\left(x(t), y_{t}\right) & \leq-\varepsilon\|x(t)\|^{2}
\end{aligned}
$$

for some $\varepsilon>0$, then the systems described by (1)-(2) is asymptotically stable.

\section{Stability CONDition OF DIE}

In Proposition 2, IOS of (2) is a necessary condition for the stability of the whole systems (1)-(2). Moreover, It is well known that IOS of (2) is equivalent to the stability of DIE presented by

$$
y(t)=\sum_{i=1}^{N+M} D_{i} y\left(t-r_{i}\right)+\sum_{i=1}^{N} M_{i} \int_{-r_{i}}^{0} y(t+\theta) \mathrm{d} \theta .
$$

If $M_{i}=0(i=1,2, \ldots N)$ [9] gave a sufficient condition for stability of the difference equations described by

$$
y(t)=\sum_{i=1}^{N+M} D_{i} y\left(t-r_{i}\right)
$$

Namely, the difference equation (7) is delay-independent exponentially stable, if there exist symmetric positive definite matrices $S_{k}, i=1,2, \ldots, N+M$ such that

$$
\operatorname{diag}\left(\begin{array}{llll}
S_{1} & S_{2} & \cdots & S_{N+M}
\end{array}\right)-D^{T}\left(\begin{array}{l}
\sum_{k=1}^{N+M} S_{k} \\
S^{2}
\end{array}\right) D>0
$$

where $D=\left(\begin{array}{llll}D_{1} & D_{2} & \cdots & D_{N+M}\end{array}\right)$.

For given any initial function $\phi \in \mathcal{P C}$, we denotes the unique solution of (6) in $y(t, \phi)$ and $y_{t}(\phi)=\{y(t+\theta, \phi) \mid \theta \in[-r, 0)\}$ stands for the partial trajectory of the system (6). For the exponential stability of (6), we introduce the Lyapunov-Krasovskii conditions from [13].

Proposition 3: Supposed the difference equation (7) is delay-independent exponentially stable. If there a functional $v: \mathcal{P C} \rightarrow \mathbb{R}$ such that $t \rightarrow v\left(y_{t}(\phi)\right)$ is differentiable and the following conditions hold:

$$
\begin{gathered}
\alpha_{1}\|\phi\|_{L_{2}}^{2} \leq v(\phi) \leq \alpha_{2}\|\phi\|_{L_{2}}^{2}, \\
\dot{v}\left(y_{t}(\phi)\right) \leq-\beta\left\|y_{t}(\phi)\right\|_{L_{2}}^{2},
\end{gathered}
$$

for some $\alpha_{2}>\alpha_{1}>0$ and $\beta>0$, then the difference-integral equation (6) is exponentially stable.

By Proposition 3, we will give a stability condition of (6) based on LMI in the following lemma.

Lemma 4: DIE (6) is exponentially stable if there exist positive definite matrices $X_{i} \in \mathbb{R}^{m}, i=1,2, \ldots, N+M$, and $Y_{j} \in \mathbb{R}^{m}, j=1,2, \ldots, N$, such that LMI

$$
\left(\begin{array}{ccc}
X & 0 & D^{T} \mathcal{G}(X, Y) \\
0 & Y & M^{T} \mathcal{G}(X, Y) \\
\mathcal{G}(X, Y) D & \mathcal{G}(X, Y) M & \mathcal{G}(X, Y)
\end{array}\right)>0
$$

is satisfied, where

$$
\begin{aligned}
X & =\operatorname{diag}\left(\begin{array}{llll}
X_{1} & X_{2} & \cdots & X_{N+M}
\end{array}\right), \\
Y & =\operatorname{diag}\left(\begin{array}{llll}
\frac{Y_{1}}{r_{1}} & \frac{Y_{2}}{r_{2}} & \cdots & \frac{Y_{N}}{r_{N}}
\end{array}\right), \\
\mathcal{G}(X, Y) & =\sum_{i=1}^{N+M} X_{i}+\sum_{j=1}^{N} r_{j} Y_{j}, \\
D & =\left(\begin{array}{llll}
D_{1} & D_{2} & \cdots & D_{N+M}
\end{array}\right), \\
M & =\left(\begin{array}{llll}
M_{1} & M_{2} & \cdots & M_{N}
\end{array}\right) .
\end{aligned}
$$


Proof: In order to save space, we just write three steps of the proof process. (i)Apply Schur's compensation to (9). (ii) Choose Lyapunov functional as

$$
\begin{aligned}
v\left(t, y_{t}\right) & =\sum_{i=1}^{N+M} \int_{-r_{i}}^{0} y^{T}(t+\theta) X_{i} y(t+\theta) \mathrm{d} \theta \\
& +\sum_{j=1}^{N} \int_{-r_{j}}^{0} \mathrm{~d} \theta \int_{t+\theta}^{t} y^{T}(\tau) Y_{j} y(\tau) \mathrm{d} \tau .
\end{aligned}
$$

(iii)The above Lyapunov functional satisfies the first condition of Proposition 3. Use the Jensen inequality in the derivative of $v$ to obtain the second condition.

Remark 5: Based on the characteristic function associated to (6), [13] gave a exponentially stability condition

$$
\sum_{i=1}^{N+M}\left\|D_{i}\right\|+r \max _{1 \leq i \leq N}\left\|M_{i}\right\|<1 .
$$

The example 2 in [13] showed that although above inequality could be easily verity, it may be pretty conservative. The example also can illustrated that (8) is less conservative than the condition $\sum_{i=1}^{N+M}\left\|D_{i}\right\|<1$ for (7).

\section{Stability Conditions of the Systems}

\section{A. Derivative of $L K F$}

In this section, we consider the stability problem of the systems (1)-(2) with the initial condition. In order to do a simple denotation in the following, define a real number sequence

$$
\mathcal{X}(i)=\left\{\begin{array}{l}
1, i=1,2, \ldots, N \\
0, i=N+1, N+2, \ldots, N+M
\end{array}\right.
$$

and a matrix sequence

$$
\overline{\mathcal{S}}(i)=\left\{\begin{array}{l}
S_{i}\left(-r_{i}\right), i=1,2, \ldots, N \\
\bar{S}_{i}, i=N+1, N+2, \ldots, N+M .
\end{array}\right.
$$

Taking derivative to $V$ along the system trajectory, we have

$$
\begin{aligned}
& \dot{V}\left(x(t), y_{t}\right)=-\sum_{i=0}^{N+M} \sum_{j=0}^{N+M} z_{i}^{T}(t) \bar{\Delta}_{i j} z_{j}(t) \\
& +2 \sum_{i=1}^{N} x^{T}(t) \int_{-r_{i}}^{0}\left[\Pi_{0 i}(\eta)-\dot{Q}_{i}(\eta)\right] y(t+\eta) \mathrm{d} \eta \\
& +2 \sum_{i=1}^{N} \sum_{j=1}^{N+M} z_{j}^{T}(t) \int_{-r_{i}}^{0} \Pi_{j i}(\eta) y(t+\eta) \mathrm{d} \eta \\
& +\sum_{i=1}^{N} \sum_{j=1}^{N} \int_{-r_{i}}^{0} \int_{-r_{j}}^{0} y^{T}(t+\xi) \Lambda_{i j}(\xi, \eta) y(t+\eta) \mathrm{d} \eta \mathrm{d} \xi \\
& -\sum_{i=1}^{N} \int_{-r_{i}}^{0} y^{T}(t+\eta) \dot{S}_{i}(\eta) y(t+\eta) \mathrm{d} \eta
\end{aligned}
$$

where

$$
z_{i}(t)= \begin{cases}x(t), & i=0 \\ y\left(t-r_{i}\right), & 1 \leq i \leq N+M\end{cases}
$$

$$
\begin{gathered}
\bar{\Delta}_{00}=\quad-A^{T} P-P A-C^{T} \bar{S}_{\Sigma}(0) C \\
\quad-\bar{Q}_{\Sigma}(0) C-C^{T} \bar{Q}_{\Sigma}^{T}(0), \\
\bar{\Delta}_{0 i}=\quad-P B_{i}-\bar{Q}_{\Sigma}(0) D_{i} \\
\quad-C^{T} \bar{S}_{\Sigma}(0) D_{i}+\mathcal{X}(i) Q_{i}\left(-r_{i}\right), \\
\bar{\Delta}_{i i}=\quad \overline{\mathcal{S}}(i)-D_{i}^{T} \bar{S}_{\Sigma}(0) D_{i}, \\
1 \leq \quad i \leq N+M \\
\bar{\Delta}_{i j} \quad=\quad-D_{i}^{T} \bar{S}_{\Sigma}(0) D_{j}, \\
\quad 1 \leq \quad i, j \leq N+M(i \neq j), \\
\Pi_{0 i}=P H_{i}+A^{T} Q_{i}(\eta)+C_{j}^{T} \bar{S}_{\Sigma}(0) M_{i} \\
+C^{T} \bar{R}_{i \Sigma}^{T}(\eta, 0)+\bar{Q}_{\Sigma}(0) M_{i}, 1 \leq i \leq N, \\
\Pi_{j i}(\eta)=B_{j} Q_{i}(\eta)+D_{j}^{T} \bar{R}_{i \Sigma}^{T}(\eta, 0) \\
+D_{j}^{T} \bar{S}_{\Sigma}(0) M_{i}-\mathcal{X}(j) R_{j i}^{T}\left(\eta,-r_{j}\right), \\
1 \leq j \leq N^{\prime}+M, 1 \leq i \leq N ; \\
\Lambda_{i j}(\xi, \eta)=H_{i}^{T} Q_{j}(\eta)+Q_{i}^{T}(\xi) H_{j}+M_{i}^{T} \bar{S}_{\Sigma}(0) M_{j} \\
+M_{i}^{T} \bar{R}_{j \Sigma}^{T}(\eta, 0)+\bar{R}_{i \Sigma}(\xi, 0) M_{j} \\
-\frac{\partial R_{i j}(\xi, \eta)}{\partial \xi}-\frac{\partial R_{i j}(\xi, \eta)}{\partial \eta}, 1 \leq i, j \leq N .
\end{gathered}
$$

with

$$
\begin{aligned}
\bar{Q}_{\Sigma}(0) & =\sum_{k=1}^{N} Q_{k}(0), \quad \bar{R}_{i \Sigma}(\eta, 0)=\sum_{k=1}^{N} R_{i k}(\eta, 0), \\
\bar{S}_{\Sigma}(0) & =\sum_{k=1}^{N} S_{k}(0)+\sum_{k=N+1}^{N+M} \bar{S}_{k} .
\end{aligned}
$$

Using Proposition 2, we can conclude that the system is asymptotically stable if (9) holds and the LKF and its derivative satisfy (4) and (5) for some $\varepsilon>0$.

\section{B. Discretization of $L K F$}

Similar to [8], in the following we will restrict the functions $Q_{i}, R_{i j}$ and $S_{i}$ to be piecewise linear. Specially, divide the interval $\left[-r_{i}, 0\right], i=1,2, \ldots, N$ into $N_{i}$ smaller intervals $\mathcal{I}_{i p}=$ $\left[\theta_{i p}, \theta_{i, p-1}\right]$ of equal length $h_{i}=r_{i} / N_{i}$, where $\theta_{i p}=-p h_{i}$.

Let

$$
\begin{array}{r}
Q_{i}^{(p)}(\alpha) \stackrel{\text { def }}{=} Q_{i}\left(\theta_{i p}+\alpha h_{i}\right)=(1-\alpha) Q_{i}^{p}+\alpha Q_{i}^{p-1} ; \\
S_{i}^{(p)}(\alpha) \stackrel{\text { def }}{=} S_{i}\left(\theta_{i p}+\alpha h_{i}\right)=(1-\alpha) S_{i}^{p}+\alpha S_{i}^{p-1} ; \\
R_{i j}^{(p q)}(\alpha, \beta) \stackrel{\text { def }}{=} R_{i j}\left(\theta_{i p}+\alpha h_{i}, \theta_{j q}+\beta h_{j}\right) \\
=\left\{\begin{array}{c}
(1-\alpha) R_{i j}^{p q}+\beta R_{i j}^{p-1, q-1}+(\alpha-\beta) R_{i, j}^{p-1, q} \\
(1-\beta) R_{i j}^{p q}+\alpha R_{i j}^{p-1, q-1}+(\beta-\alpha) R_{i j}^{p, q-1} \\
\text { for } \alpha \geq \beta ;
\end{array}\right.
\end{array}
$$

for $0 \leq \alpha, \beta \leq 1, p=1,2, \ldots, N_{i}, q=1,2, \ldots, N_{j} ; i=$ $1,2, \ldots, N, j=1,2, \ldots, N$.

This choice allows us to write the stability conditions (4) and (5) in the form of LMIs. The idea is very similar to that of [10]. 
Remark 6: Note that the discretized approach above for the system with multple delays is different to the one in [8], where the corresponding discretization was carried out in the delay-parameter space $O r_{N} r_{N}$. In this paper, however, this discretization was derived in the delay-parameter space $O r_{1} r_{2} \cdots r_{N}$ which is first presented in [12].

\section{LKF Condition}

The following lemma specifies the conditions for (4).

Lemma 7: The LKF $V$ defined in (3), with piecewise linear matrices $Q_{i}, R_{i j}$, and $S_{i}$ as defined in (11) to (13), satisfies (4) if

$$
\begin{aligned}
& S_{i}^{p}>0, p=1,2, \ldots, N_{i}, i=1,2, \ldots, N, \\
& \bar{S}_{k} \geq 0, k=N+1, N+2, \ldots, M+N,
\end{aligned}
$$

and

$$
\left(\begin{array}{cc}
P & \mathcal{Q} \\
\mathcal{Q}^{T} & \mathcal{R}+\mathcal{S}
\end{array}\right)>0
$$

are satisfied, where

$$
\begin{aligned}
& \mathcal{Q}=\left(\begin{array}{llll}
\tilde{Q}_{1} & \tilde{Q}_{2} & \cdots & \tilde{Q}_{N}
\end{array}\right), \\
& \tilde{Q}_{i}=\left(\begin{array}{llll}
Q_{i}^{0} & Q_{i}^{1} & \cdots & Q_{i}^{N_{i}}
\end{array}\right), \\
& \mathcal{R}=\left(\tilde{R}_{i j}\right)_{i=1 \rightarrow N}^{j=1 \rightarrow N} \quad\left(\tilde{R}_{i j}^{T}=\tilde{R}_{j i}\right), \\
& \tilde{R}_{i j}=\left(R_{i j}^{p q}\right)_{p=1 \rightarrow N_{i}}^{q=1 \rightarrow N_{j}}, \\
& \mathcal{S}=\operatorname{diag}\left(\begin{array}{cccc}
\tilde{S}_{1} & \tilde{S}_{2} & \cdots & \tilde{S}_{N}
\end{array}\right), \\
& \tilde{S}_{i}=\operatorname{diag}\left(\begin{array}{cccc}
\frac{1}{h_{i}} S_{i}^{0} & \frac{1}{h_{i}} S_{i}^{1} & \cdots & \frac{1}{h_{i}} S_{i}^{N_{i}}
\end{array}\right) .
\end{aligned}
$$

Proof: Since $V_{2}$ in (3) is positive semi-definite, we only need to show that $V_{1}$ is positive definite. This is similar to the one discussed in [10].

\section{Derivative Condition of $L K F$}

The next lemma established the conditions for (5). For simplicity of expression, define a matrix sequence

$$
\mathcal{S}(i)=\left\{\begin{array}{l}
S_{i}^{N_{i}}, i=1,2, \ldots, N \\
\bar{S}_{i}, i=N+1, N+2, \ldots, N+M .
\end{array}\right.
$$

Let

$$
\begin{aligned}
\bar{Q}_{\Sigma}^{0} & =\sum_{k=1}^{N} Q_{k}^{0}, \quad \bar{R}_{i \Sigma}^{p 0}=\sum_{k=1}^{N} R_{i k}^{p 0} \\
\bar{S}_{\Sigma}^{0} & =\sum_{k=1}^{N} S_{k}^{0}+\sum_{k=N+1}^{N+M} \bar{S}_{k} .
\end{aligned}
$$

Lemma 8: The derivative $\dot{V}$ of the LKF in (10), with $Q_{i}$, $S_{i}$ and $R_{i j}(i, j=1,2, \ldots, N)$ piecewise linear as expressed in (11) to (13), satisfies (5) if there exists a real matrix $W=W^{T}$ such that

$$
\begin{gathered}
\left(\begin{array}{ccc}
\bar{\Delta} & -Y^{s} & -Y^{a} \\
-Y^{s T} & \Gamma-M^{T} S_{\Sigma}^{0} M & -\digamma^{a} \\
-Y^{a T} & -\digamma^{a} & 3\left(S_{d}-W\right)
\end{array}\right)>0 \\
\left(\begin{array}{cc}
W & R_{d a} \\
R_{d a}^{T} & W
\end{array}\right)>0
\end{gathered}
$$

where

$$
\begin{gathered}
\Gamma=R_{d}+S_{d}-W-\left(\digamma^{s}+\digamma^{s T}\right), \\
\bar{\Delta}=\left(\bar{\Delta}_{i j}\right)_{i=0 \rightarrow N+M}^{j=0 \rightarrow N+M} \quad\left(\bar{\Delta}_{i j}^{T}=\bar{\Delta}_{j i}\right),
\end{gathered}
$$

$$
\begin{aligned}
\bar{\Delta}_{00} & =-A^{T} P-P A-C^{T} S_{\Sigma}^{0} C-\bar{Q}_{\Sigma}^{0} C-C^{T} \bar{Q}_{\Sigma}^{0 T}, \\
\bar{\Delta}_{0 i} & =-P B_{i}-C^{T} S_{\Sigma}^{0} D_{i}-\bar{Q}_{\Sigma}^{0} D_{i}+\mathcal{X}(i) Q_{i}^{N_{i}}, \\
1 & \leq i \leq N+M ; \\
\bar{\Delta}_{i j} & =-D_{i}^{T} S_{\Sigma}^{0} D_{j}(i \neq j), \quad 1 \leq i, j \leq M+N, \\
\bar{\Delta}_{i i} & =\mathcal{S}(i)-D_{i}^{T} S_{\Sigma}^{0} D_{i}, \quad 1 \leq i \leq N+M ;
\end{aligned}
$$

$$
\begin{aligned}
& S_{d}=\operatorname{diag}\left(\begin{array}{llll}
S_{d 1} & S_{d 2} & \cdots & S_{d N}
\end{array}\right), \\
& S_{d i}=\operatorname{diag}\left(\begin{array}{cccc}
S_{d i}^{1} & S_{d i}^{2} & \cdots & S_{d i}^{N_{i}}
\end{array}\right), \\
& S_{d i}^{p}=\frac{1}{h_{i}^{2}}\left(S_{i}^{p-1}-S_{i}^{p}\right)
\end{aligned}
$$

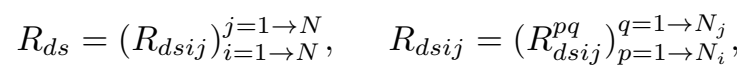

$$
\begin{aligned}
& R_{d s i j}^{p q}=\frac{1}{2}\left(\frac{1}{h_{i}}+\frac{1}{h_{j}}\right)\left(R_{i j}^{p-1, q-1}-R_{i j}^{p q}\right) \\
& +\frac{1}{2}\left(\frac{1}{h_{j}}-\frac{1}{h_{i}}\right)\left(R_{i j}^{p, q-1}-R_{i j}^{p-1, q}\right) ; \\
& R_{d a}=\left(R_{d a i j}\right)_{i=1 \rightarrow N}^{j=1 \rightarrow N}, \quad R_{d a i j}=\left(R_{d a i j}^{p q}\right)_{p=1 \rightarrow N_{i}}^{q=1 \rightarrow N_{j}}, \\
& R_{d a i j}^{p q}=\frac{1}{2}\left(\frac{1}{h_{j}}-\frac{1}{h_{i}}\right)\left(R_{i j}^{p-1, q-1}-R_{i j}^{p-1, q}-R_{i j}^{p, q-1}+R_{i j}^{p q}\right) ; \\
& Y^{s}=\left(Y_{i j}^{s}\right)_{i=0 \rightarrow N+M}^{j=1 \rightarrow N}, \\
& Y_{i j}^{s}=\left(\begin{array}{llll}
Y_{i j}^{s 1} & Y_{i j}^{s 2} & \cdots & Y_{i j}^{s N_{j}}
\end{array}\right), \\
& Y_{0 i}^{s p}=P H_{i}+\bar{Q}_{\Sigma}^{0} M_{i}+C^{T} S_{\Sigma}^{0} M_{i}-\frac{1}{h_{i}}\left(Q_{i}^{p-1}-Q_{i}^{p}\right) \\
& +\frac{1}{2} A^{T}\left(Q_{i}^{p}+Q_{i}^{p-1}\right)+\frac{1}{2} C^{T}\left(\bar{R}_{i \Sigma}^{p, 0 T}+\bar{R}_{i \Sigma}^{p-1,0 T}\right), \\
& Y_{j i}^{s p}=\frac{1}{2} B_{j}^{T}\left(Q_{i}^{p}+Q_{i}^{p-1}\right)+\frac{1}{2} D_{j}^{T}\left(\bar{R}_{i \Sigma}^{p, 0 T}+\bar{R}_{i \Sigma}^{p-1,0 T}\right) \\
& -\frac{1}{2} \mathcal{X}(j)\left(R_{j i}^{p, N_{j} T}+R_{j i}^{p-1, N_{j} T}\right)+D_{j}^{T} S_{\Sigma}^{0} M_{i}, \\
& 1 \leq i \leq N, 1 \leq j \leq N+M \\
& Y^{a}=\left(Y_{i j}^{a}\right)_{i=0 \rightarrow N+M}^{j=1 \rightarrow N}, \\
& Y_{i j}^{a}=\left(\begin{array}{llll}
Y_{i j}^{a 1} & Y_{i j}^{a 2} & \ldots & Y_{i j}^{a N_{j}}
\end{array}\right), \\
& Y_{0 i}^{a p}=\frac{1}{2} A^{T}\left(Q_{i}^{p}-Q_{i}^{p-1}\right)+\frac{1}{2} C^{T}\left(\bar{R}_{i \Sigma}^{p, 0 T}-\bar{R}_{i \Sigma}^{p-1,0 T}\right), \\
& Y_{j i}^{a p}=\frac{1}{2} B_{j}^{T}\left(Q_{i}^{p}-Q_{i}^{p-1}\right)+\frac{1}{2} D_{j}^{T}\left(\bar{R}_{i \Sigma}^{p, 0 T}-\bar{R}_{i \Sigma}^{p-1,0 T}\right) \\
& -\frac{1}{2} \mathcal{X}(j)\left(R_{j i}^{p, N_{j} T}-R_{j i}^{p-1, N_{j} T}\right), \\
& 1 \leq j \leq N+M, 1 \leq i \leq N \text {; } \\
& \digamma^{s}=\left(\digamma_{i j}^{s}\right)_{i=1 \rightarrow N}^{j=1 \rightarrow N}, \quad \digamma_{i j}^{s}=\left(\digamma_{i j}^{s p q}\right)_{i=0 \rightarrow N_{i}}^{j=1 \rightarrow N_{j}},
\end{aligned}
$$




$$
\begin{gathered}
\digamma_{i j}^{s p q}=\frac{1}{2} H_{i}^{T}\left(Q_{j}^{q}+Q_{j}^{q-1}\right)+\frac{1}{2} M_{i}^{T} \sum_{k=1}^{N}\left(R_{j k}^{q, 0 T}+R_{j k}^{q-1,0 T}\right), \\
\digamma^{a}=\left(\digamma_{i j}^{a}\right)_{i=1 \rightarrow N}^{j=1 \rightarrow N}, \quad \digamma_{i j}^{a}=\left(\digamma_{i j}^{a p q}\right)_{i=0 \rightarrow N_{i}}^{j=1 \rightarrow N_{j}}, \\
\digamma_{i j}^{a p q}=\frac{1}{2} H_{i}^{T}\left(Q_{j}^{q}-Q_{j}^{q-1}\right)+\frac{1}{2} M_{i}^{T}\left(\bar{R}_{j \Sigma}^{q, 0 T}-\bar{R}_{j \Sigma}^{q-1,0 T}\right) .
\end{gathered}
$$

Proof: The proof is similar to that of Proposition 5 in [8].

Remark 9: It is noticed that (15) in Lemma 8 is evidently different with (16) of Theorem 3 in [11] by the dimension of $\bar{\triangle}, Y^{s}$ and $Y^{a}$, the term $\mathcal{S}(i)$ in $\bar{\triangle}_{i i}$, the multiplier $\mathcal{X}(i)$ in $\bar{\triangle}_{0 i}, Y_{j i}^{s p}$ and $Y_{j i}^{a p}$.

\section{Stability of the Whole Systems}

Combining Lemma 4, 7 and 8 together and applying Schur's compensation we obtain the stability condition of the systems (1)-(2) as follows.

Theorem 10: The system expressed by (1)-(2) is uniformly asymptotically stable if there exist $n$ by $n$ matrix $P=P^{T}, n$ by $m$ matrices $Q_{i}^{p}, m$ by $m$ matrices $R_{i j}^{p q}, S_{i}^{p}, p=1,2, \ldots, N_{i}, q=$ $1,2, \ldots, N_{j}, i=1,2, \ldots, N, j=1,2, \ldots, N$; and $m$ by $m$ matrix $W=W^{T}, X_{i}, i=1,2, \ldots, N+M, Y_{j}, j=1,2, \ldots, N$,such that (9) (14) (15) and

$$
\left(\begin{array}{cccc}
\Delta & -Y^{s} & -Y^{a} & Z^{T} S_{\Sigma}^{0} \\
-Y^{s T} & \Gamma & -\digamma^{a} & M^{T} S_{\Sigma}^{0} \\
-Y^{a T} & -\digamma^{a T} & 3\left(R_{d}-W\right) & 0 \\
S_{\Sigma}^{0} Z^{T} & S_{\Sigma}^{0} M & 0 & S_{\Sigma}^{0}
\end{array}\right)>0
$$

are satisfied, with the notation defined in (15) to (15), (18) to (18) and

$$
\Delta=\left(\Delta_{i j}\right)_{i=0 \rightarrow N+M}^{j=0 \rightarrow N+M}
$$

$\Delta_{00}=-A^{T} P-P A-\sum_{k=1}^{N}\left(Q_{k}^{0} C+C^{T} Q_{k}^{0 T}\right)$

$\Delta_{0 i}=-P B_{i}-\sum_{k=1}^{N} Q_{k}^{0} D_{i}+\mathcal{X}(i) Q_{i}^{N_{i}}, 1 \leq i \leq N+M ;$

$\Delta_{i j}=0(i \neq j), 1 \leq i, j \leq M+N$,

$\Delta_{i i}=\mathcal{S}(i), 1 \leq i \leq M+N$;

and

$$
Z=\left(\begin{array}{ccccc}
C & D_{1} & D_{2} & \cdots & D_{N+M}
\end{array}\right) .
$$

\section{NUMERICAL EXAMPLES}

Example 1: Consider a system with two delays

$$
\begin{aligned}
\dot{x}(t)= & A x(t)+B y_{2}\left(t-r_{2}\right)+H \int_{-r_{1}}^{0} y_{1}(t+\theta) \mathrm{d} \theta, \\
y_{1}(t)= & C_{1} x(t)+K_{1} \int_{-r_{1}}^{0} y_{1}(t+\theta) \mathrm{d} \theta, \\
y_{2}(t)= & C_{2} x(t)+D_{1} y_{1}\left(t-r_{1}\right)+D_{2} y_{2}\left(t-r_{2}\right) \\
& -K_{2} \int_{-r_{1}}^{0} y_{1}(t+\theta) \mathrm{d} \theta
\end{aligned}
$$

where

$$
\begin{aligned}
& A=\left(\begin{array}{cccccc}
0 & 0.5 & 0 & 0 & 0 & 0 \\
-0.5 & -1 & 0 & 0 & 0 & 0 \\
1 & 1 & -2 & 0 & 0 & 0 \\
0 & 0 & 0 & -0.9 & 0 & 0 \\
0 & 0 & 1 & 0 & -1 & 0 \\
0 & 0 & 0 & 0 & 1 & -1
\end{array}\right), \\
& B=\left(\begin{array}{cc}
0 & 0 \\
0 & 0 \\
-2 & 0 \\
-1 & -1.45 \\
0 & 0 \\
0 & 0
\end{array}\right), \quad H=\left(\begin{array}{c}
0.25 \\
-0.5 \\
2.5 \\
0 \\
-1 \\
0
\end{array}\right) \\
& C_{1}=\left(\begin{array}{llllll}
0 & 1 & 0 & 0 & 0 & 0
\end{array}\right), \\
& C_{2}=\left(\begin{array}{cccccc}
0.2 & 0 & 1 & 0 & 0 & 0 \\
0 & 0 & 0 & 1 & 0 & 0
\end{array}\right) \\
& D_{1}=\left(\begin{array}{c}
0.2 \\
0
\end{array}\right), D_{2}=0.5 I_{2}, K_{1}=0.5, K_{2}=\left(\begin{array}{c}
-1 \\
0
\end{array}\right) \text {. }
\end{aligned}
$$

This system come from [10], where it is given that the system is exponentially stable if and only if $r_{1} \in[0,2 \pi)$ and $r_{2} \in[0,4.7388)$. Although the above system and the example in [10] have the same characteristic equations, their stability is not equivalent. In fact, the additional dynamics

$$
\operatorname{det}\left(I+K_{1} \frac{1-e^{-r_{1} s}}{s}\right)=0
$$

is introduced in the difference-integral equation that the stability is a necessary condition for the whole systems, so that the stable region of $r_{1}$ is determined by both the above equation and the characteristic equations. Noting that the additional dynamics is the characteristic equation of the integral equation

$$
y(t)+0.5 \int_{-r_{1}}^{0} y(t+\sigma) \mathrm{d} \sigma=0 .
$$

Using the condition of Example 1 in [13], we obtained that (20) is exponentially stable if and only if $r_{1} \in[0,2)$. Connecting the stable interval $[0,2 \pi)$ of $r_{1}$, we obtained that the whole systems is exponentially stable if and only if $r_{1} \in[0,2)$ and $r_{2} \in[0,4.7388)$.

Applied Theorem 10 of this paper, for four given ratios $1 / \sqrt{5}, 1 / \sqrt{2}, \sqrt{2}$ and $\sqrt{5}$ of $r_{1} / r_{2}$, the upper bound of the delay $r_{2}$ are computed by using MATLAB program with a bisection process, and the results are listed in the following table.

\begin{tabular}{ccccc}
\hline$r_{1} / r_{2}$ & $1 / \sqrt{5}$ & $1 / \sqrt{2}$ & $\sqrt{2}$ & $\sqrt{5}$ \\
\hline$\left(N_{1}, N_{2}\right)$ & $(1,2)$ & $(1,2)$ & $(2,1)$ & $(2,1)$ \\
$r_{2 \max }$ & 4.4721 & 2.8284 & 1.4142 & 0.8944 \\
\hline
\end{tabular}

From above table we are not difficult to find, when the ratios of $r_{1} / r_{2}$ as $1 / \sqrt{5}, 1 / \sqrt{2}, \sqrt{2}$ and $\sqrt{5}$, the supremum of $r_{1}$ is 2 . In fact, if the ratios of $r_{1} / r_{2}$ is more than $2 / 4.7388$, the maximum estimate of $r_{2}$ is completely restricted by the supremum 2 of $r_{1}$. However, for some fixed $r_{1} \in[0,2)$ or setting $r_{1} / r_{2} \in$ 
$[0,2 / 4.7388)$, we can calculate that the maximum estimate of $r_{2}$ can approximate the analytical limit. In the following we gave the results for $r_{1}=1.9999$ and $r_{1} / r_{2}=0.4$.

\begin{tabular}{ccccc}
\hline$r_{1}=1.9999$ & & & & \\
\hline$\left(N_{1}, N_{2}\right)$ & $(1,2)$ & $(2,3)$ & $(2,4)$ & Analytical \\
$r_{2 \max }$ & 4.7354 & 4.7381 & 4.7385 & 4.7388 \\
\hline$r_{1} / r_{2}=0.4$ & & & & \\
\hline$\left(N_{1}, N_{2}\right)$ & $(1,2)$ & $(1,3)$ & $(2,4)$ & Analytical \\
$r_{2 \max }$ & 4.7354 & 4.7381 & 4.7385 & 4.7388 \\
\hline
\end{tabular}

Example 2: Consider a two-dimensional system

$$
\begin{aligned}
\dot{x}(t)-D_{0} \dot{x}(t-r)= & A_{0} x(t)+B_{0} x(t-r) \\
& +C_{0} \int_{-\tau}^{0} x(t+s) \mathrm{d} s
\end{aligned}
$$

with

$$
\begin{aligned}
A_{0} & =\left(\begin{array}{cc}
-a_{1} & 0 \\
0 & -a_{2}
\end{array}\right), B_{0}=\left(\begin{array}{cc}
b_{1} & b_{2} \\
-b_{2} & b_{1}
\end{array}\right), \\
C_{0} & =\left(\begin{array}{cc}
c_{1} & c_{2} \\
-c_{2} & c_{2}
\end{array}\right) .
\end{aligned}
$$

[14] and [15] discussed the stability of system for all $r$ and allowable $\tau$. For the different parameters, it is verified by three cases.

C1: $D_{0}=0, a_{1}=a_{2}=1.5, b_{1}=b_{2}=1, c_{1}=1, c_{2}=0.5$.

C2: $D_{0}=0, a_{1}=2, a_{2}=15, b_{1}=1, b_{2}=3, c_{1}=1, c_{2}=$ 0.5 .

C3: $D_{0}=\left(\begin{array}{cc}-0.2 & 0 \\ 0.2 & -0.1\end{array}\right), a_{1}=2, a_{2}=15, b_{1}=1 ; b_{2}=$ $3, c_{1}=1, c_{2}=0.5$.

The system may be transformed into a standard coupled equation form as (1)-(2)

$$
\begin{aligned}
& \dot{\tilde{x}}(t)=A \tilde{x}(t)+B \tilde{y}(t-r)+M \int_{-\tau}^{0} \tilde{y}(t+s) \mathrm{d} s, \\
& \tilde{y}(t)=\tilde{x}(t)+D_{0} \tilde{y}(t-r)-k \int_{-\tau}^{0} \tilde{y}(t+s) \mathrm{d} s .
\end{aligned}
$$

where $k$ is a adjusting parameter and $A=A_{0}+k I, B=$ $B_{0}+\left(A_{0}+k I\right) D_{0}, M=C_{0}-\left(A_{0}+k I\right) k$.

While $k=0$, applying the theorem of this paper, the stability of system are test for all $r$ and allowable $\tau$. For the above three cases of parameters, the results are listed in the following table with the results in [15] and [14] together.

\begin{tabular}{cccc}
\hline$\tau_{\max }$ & {$[14]$} & {$[15]$} & Theorem 1 for $N=2$ \\
\hline $\mathrm{C} 1$ & 0.03 & 0.07 & 0.1138 \\
$\mathrm{C} 2$ & Not & 1.1 & 1.2897 \\
$\mathrm{C} 3$ & Not & 1 & 1.2303 \\
\hline
\end{tabular}

For some fixed $k(\neq 0)$ satisfied the condition (9), and let $N=2$, the stability of system are test for all $r$ and allowable $\tau$. For the case 1 and case 2 the results is same to the one with $k=0$. For the case 3 the results are listed in the following table.

\begin{tabular}{cccccc}
\hline$k$ & -0.5 & -0.3 & -0.1 & 0.1 & 0.3 \\
\hline $\mathrm{C} 3$ & 1.2299 & 1.2308 & 1.2306 & 1.2301 & 1.2298 \\
\hline
\end{tabular}

The above data show, for the selection of suitable $k$, stability of the systems can be improved at a certain level.

\section{CONCLUSION}

The stability of a class of coupled differential-functional equations with multiple discrete and distributed delays is studied via a LKF approach. The discretized LKF method is used to render the problem into an LMI form that the dicretization only involved to divide the known delay interval. The stability condition presented is independent with unknown delays, is dependent with known delays.

\section{REFERENCES}

[1] J. K. Hale, and S. M. Verduyn Lunel, Introduction to Functional Differential Equations, Springer-Verlag, New York, 1993.

[2] V. Răsvan, "Dynamical Systems with lossless propagation and neutral functional differential equations," Proc. MTNS 98, Padoue, Italy, 1998, pp. 527-531.

[3] V. Răsvan, and S.I. Niculescu, "Oscillations in lossless propagation models: a Liapunov-Krasovskii approach," IMA Journal of Mathematical Control and Information, vol. 19, pp. 157-172, 2002.

[4] V. Răsvan, "Functional differential equations of lossless propagation and almost linear behavior. Plenary Lecture," 6th IFAC Workshop on Timedelay Systems, L'Aquila, Italy, 2006, pp. 527-531.

[5] K. Gu, and S. I. Niculescu, "Stability Analysis of Time-Delay Systems: A Lyapunov Approach," Advanced Topics in Control Systems Theory. Eds:Loría, A. Lamnabhi-Lagarrigue, F. and Panteley, E., Lecture Notes from FAP 2005, Springer, London, 2006, pp. 139-170.

[6] $\mathrm{K}$. Gu, and Y. Liu, "Lyapunov-Krasovskii functional for couple differential-functional equations," Automatica, vol. 245, pp. 798-804, 2009.

[7] K. Gu, "Stability problem of system with multiple channels," Automatica, vol. 46, pp. 743-751, 2010.

[8] K. Gu, V. L. Kharitonov, and J. Chen, Stability of Time-Delay Systems, Birkhäuser, Boston, MA, 2003.

[9] H. Li, and K. Gu, "Lyapunov-Krasovskii Functional Approach for Coupled Differential-Difference Equations with Multiple Delays," Delay Differential Equations: Recent Advances and New Directions. Eds: B. Balachandran, Kalmar-Nagy, T. and Gilsinn, D. Springer, Match 2009, pp. 1-30.

[10] H. Li, and K. Gu, "Discretized Lyapunov-Krasovskii Functional for Coupled Differential-Difference Equations with Multiple Delay Channels," Automatica, vol. 46, pp. 902-909, 2010.

[11] H. Li, "Discretized LKF approach for coupled differential-difference equations with multiple discrete and distributed delays," International Journal of Robust and Nonlinear Control, vol. 22, pp.875-891, 2012.

[12] H. Li, and K. Gu, "Discretized Lyapunov-Krasovskii Functional for Coupled Differential-Difference Equations with Multiple Delay Channels," 2008 ASME dynamic systems and control conference, Ann Arbor, MI, October 20-22, 2008, pp. 1-8.

[13] D. Melchor-Arguilar, "Exponential stabiliuty of linear continuous time systems with multiple delays," Systems \& Control Letter, vol. 62, pp. 811-818, 2013.

[14] V. Kolmanovskii, and J. P. Richard, "Stability of some linear systems with delays," IEEE Trans. Automat. Control, vol. 44, pp. 984-989, 1999.

[15] E. Fridman, "New Lyapunov-Krasovskii functionals for stability of linear retarded and neutral type systems," Systems \& Control Letters, vol. 43, pp. 309-319, 2001. 